\title{
Evaluating the Attitude of Nigerian Anatomists Towards Body Donations for Medical Education
}

\author{
Ogan Christopher Akanaku ${ }^{1}$, Odey Paul Anyiom², Ikpa James Onah ${ }^{1, ~ *, ~}$ \\ Asomugha Precious Remigius ${ }^{1}$ \\ ${ }^{1}$ Department of Human Anatomy, Cross River University of Technology (Crutech), Okuku-campus, Nigeria \\ ${ }^{2}$ Department of Anatomical Sciences, Faculty of Basic Medical Sciences, University of Calabar, Calabar, Nigeria \\ Email address: \\ c.ogan@ymail.com (O. C. Akanaku), anyiomodey100@gmail.com (O. P. Anyiom), jamesonahikpa@gmail.com (I. J. Onah), \\ onyekachiadaobi1@gmail.com (A. P. Remigius) \\ ${ }^{*}$ Corresponding author
}

\section{To cite this article:}

Ogan Christopher Akanaku, Odey Paul Anyiom, Ikpa James Onah, Asomugha Precious Remigius. Evaluating the Attitude of Nigerian Anatomists Towards Body Donations for Medical Education. Biomedical Sciences. Vol. 5, No. 4, 2019, pp. 71-75.

doi: $10.11648 /$ j.bs.20190504.16

Received: September 23, 2019; Accepted: October 12, 2019; Published: November 25, 2019

\begin{abstract}
The use of dissection in anatomy has long been considered a cornerstone in medical education irrespective of nation, racial background or medical school. Anatomy education in Nigeria is faced with the challenge of insufficient supply of cadavers. Body donation could be a possible solution to the inadequate supply of cadavers for medical education in Nigeria. Very little is known about body donation in Nigeria as well as the attitude of Nigerians towards body donation. In this study the attitude of Nigerian anatomists towards body donation was evaluated. Questionnaires were sent to anatomists in three universities in Nigeria covering three geographical zones in Nigeria. A total of fifty-eight (58) responses were obtained. The use of unclaimed bodies (58.6\%) and the use of 3D models (46.6\%) were the major solutions proposed for cadaver insufficiency. $85.5 \%$ of the participants thought that body donation campaigns could increase people's willingness to donate their body for anatomical study. Only $47 \%$ of the participants were willing to campaign for body donation while $53 \%$ of the participants were not willing to campaign. Only a few of the participants $(25 \%)$ were willing to donate their bodies for anatomical study. Their unwillingness was mainly due to religious reasons $(26.5 \%)$, culture and tradition (12\%) and also restrictions from family members $(18 \%)$. Some even had reasons they would not disclose (28.5\%). They could recommend body donation to others but will not recommend it to their relatives.
\end{abstract}

Keywords: Anatomy, Anatomical Study, Medical Education, Body Donation, Nigerian Anatomists

\section{Introduction}

The utilization of dissection in anatomical sciences has over time been viewed as a foundation in medical pedagogy regardless of country, racial foundation or medicinal school $[15,17,22]$. By learning gross anatomy, medical students get an early introduction about the structure of human body, which is the reason for understanding pathologic and clinical issues $[2,5,7,14,18]$. Hands-on understanding with regards to different strategies is essential for effective teaching and learning in anatomy [9]. The study of human structures is largely subject to what we can be seen through cadaveric dissection [8] and is gradually been compromised by the absence of cadavers to address the issues of the rising number of medical schools [21]. This study is aimed at evaluating the attitudes of Nigerian anatomist towards body donation for medical education. Absence of huge body donation projects have been believed to be purposes behind deficiency of cadaveric materials. Few students know about local body donation, this is a pointer that there is absence extensive of data, information and awareness about body donation programs as such people repulse body donation due to different factors [21].

In a Turkish report, solutions proposed showed that there ought to be increment in unclaimed bodies (77.1\%), expansion in body donation programs (78.3\%). Different 
solutions proposed were to diminish the quantity of medical students $(60.2 \%)$ and utilization of anatomical models (55.4\%). Demonstrating that body donations ought to be bolstered and campaigned for [21]. These days in Europe and North America, (80-100) \% of the available cadaver for medical study are donated. In Kenya, the greater part of the available bodies $(90 \%)$ are from unclaimed bodies [10]. In India, the anatomy act was passed in the entirety of India's states. This enables bodies to be donated by the donor and bodies to be claimed for medical studies and research if there is no claim to one's body within a 48-hour timeframe [21]. In the United States, there are many body donation programs with well-organized rules for acknowledgment or refusal of donated bodies, with medical schools holding memorial services for the donated bodies, which is to show regard for the contributors and their families $[19,16]$.

There are no reports on the assessment of awareness and willingness for body donations in Nigeria, thus recommending the requirement for broad research and campaign programs in this regard, anyway Akinola, (2011) [1] has done a broad review on the need for the need for this kind of programs and its possibilities. The circumstance in Nigeria and sub - Saharan Africa is that a formal willed body program is unavailable in most anatomy departments regardless of a deficient supply of cadavers for anatomical studies and the difficulties related with sourcing cadavers for this reason [6]. It is in this way basic for departments of anatomy in Nigerian medical schools to set up body donation projects to accomplish a standard stockpile of cadavers. The Lagos State House of Assembly passed an official bill in 2007 referred to as the Lagos State Coroner's Law, which was embraced by the State Governor [11]. This law will in a way affect the supply of cadavers to medical schools this is because section 14 of the law makes autopsy a compulsory practice for all deaths of unknown circumstances. Most bodies for dissection are gotten from hospitals, subjecting all bodies for autopsy will cause a decrease in the supply of bodies for educational purposes. Osuagwu et al., (2004) [13] has proposed that the fundamental source of bodies in Nigeria is essentially from unclaimed bodies as a result of accidents and disasters and criminal remains from executions.

\section{Methods}

The study involved fifty-eight anatomists (lecturers basically) in four (4) medical faculties which include; University of Nigeria, University of Calabar, Ahmadu Bello University and Cross River University of Technology.

The questionnaire was designed to allow the participants give "AGREE" and "DISAGREE" responses to the question as well as other comments.

The results were presented in charts in the order the questions appear on the questionnaire. The individual questions were grouped into:

1. Evaluating participants' views on the relevance of cadaveric use for anatomical pedagogy

2. Knowledge on the sources and supply of cadaver for anatomical teaching in Nigeria

3. knowledge on free will body donation as cadaveric source

4. Their affirmation to free will body donation for anatomical teaching

Analysis was done with Microsoft excel and charts were generated to represent results.

\section{Results}

To evaluate participants' views on the relevance of cadaver use for anatomical pedagogy question 1-4 was used. In response, as shown in figure 1, 96\% of the participants agreed that cadaver use for anatomical and medical training is fundamental while $4 \%$ disagreed. $98 \%$ of the participants agreed that the use of cadaver has improved knowledge over time while only $2 \%$ disagreed. $72 \%$ of the participants agreed that anatomical pedagogy will be jeopardized if cadaver use was discontinued while $28 \%$ disagreed to this $86 \%$ of the participants agreed that cadaver use for anatomical teaching be sustained while $14 \%$ of the participants disagreed.

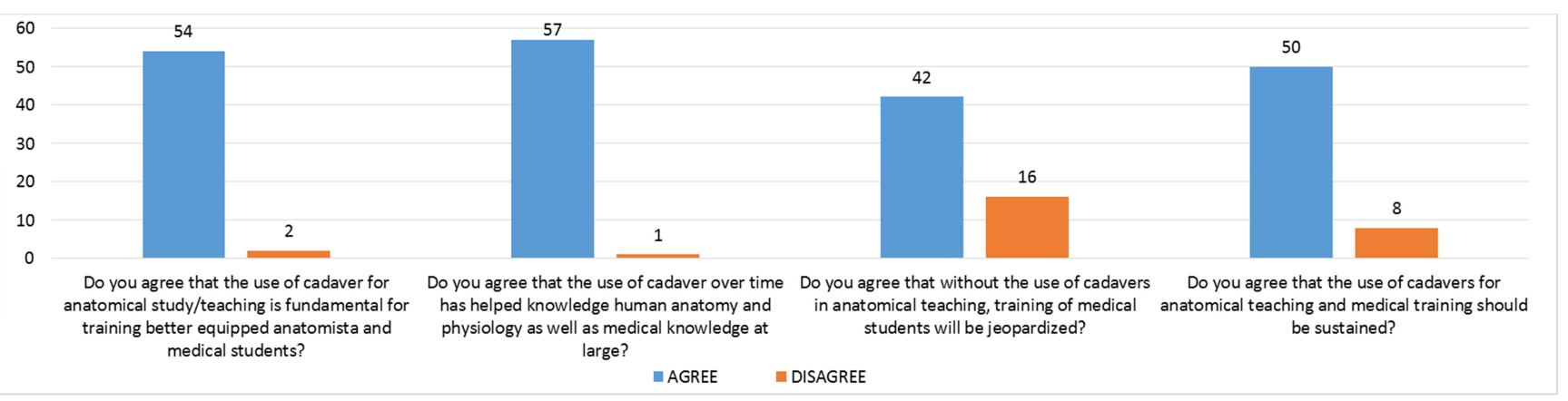

Figure 1. Evaluation of participant's views on the relevance of cadaver use for anatomical pedagogy.

In response to their knowledge on the sources and supply of cadaver for anatomical teaching in Nigeria (Questions 5-8), 84\% of the participants affirmed to knowledge of unclaimed bodies as main source of cadaver while $16 \%$ responded negatively. $67 \%$ of the participants agreed that there was cadaver supply shortage while $33 \%$ of the participant disagreed. $68 \%$ of the participants thought that the reduced supply has affected the quality of anatomical pedagogy while $32 \%$ of the participant did not think that the reduced supply has affected the quality of anatomical study. $82 \%$ of the participants agreed that it will be difficult to sustain anatomical teaching and study 
if cadaveric supply continues to decrease while $18 \%$ of the participants disagreed to this. It was observed that participants are well knowledgeable on the sources and supply of cadavers, suggesting that unclaimed bodies are the most known and most reliable source of cadavers. as shown on figure 2.

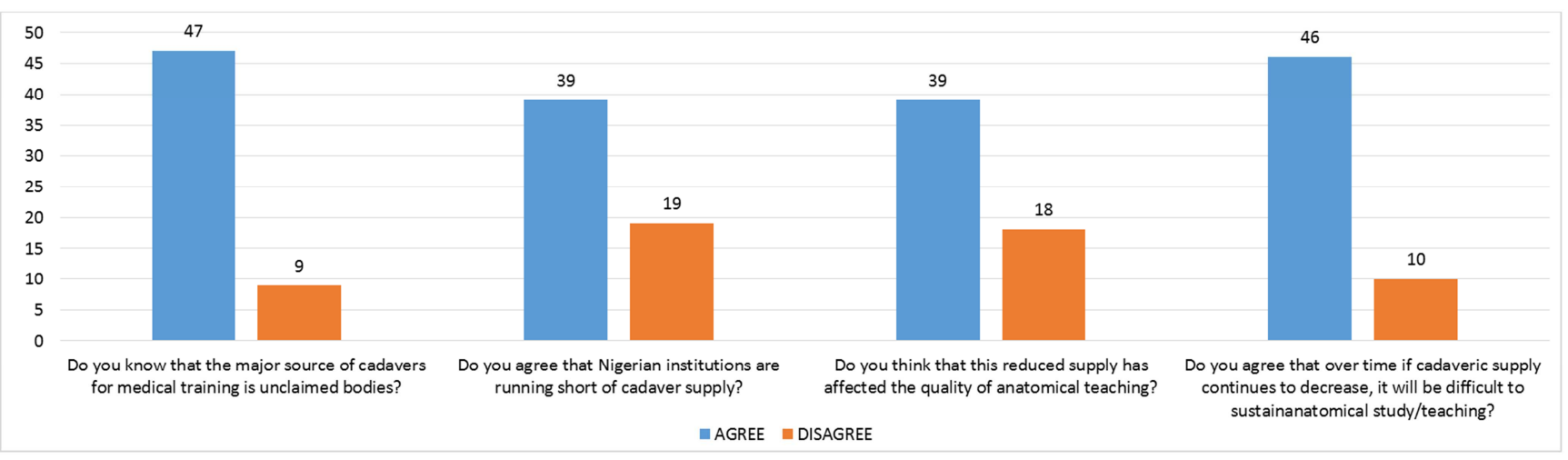

Figure 2. Evaluation of participants' knowledge on the sources and supply of cadaver for anatomical teaching in Nigeria.

Questions 10-12 were used to evaluate the participants' knowledge on free- will body donation as cadaveric source (figure 3) $72 \%$ of the participants have heard of body donation while $28 \%$ have not. $76 \%$ of the participants agreed that dead bodies that would have been burnt or buried are useful in medical schools for anatomical teaching. $71 \%$ of the participants agreed that donated bodies will help the living learn about the human body while $29 \%$ did not agree. all the participants (100\%) agreed that free-will body donation is another way by which cadaveric supply for medical pedagogy can be boosted (figure 3 ).

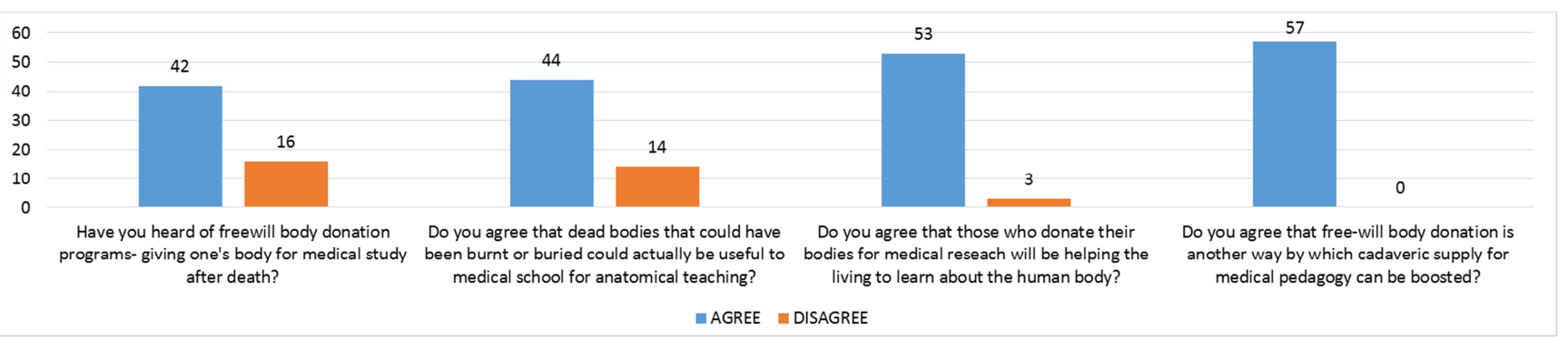

Figure 3. Evaluation of participants' knowledge on free- will body donation as cadaveric source.

Questions 13-19 were used in evaluating their willingness to free will body donation for anatomical pedagogy. In response, $85.5 \%$ of the participants thought that a campaign on body donation could increase people's willingness to donate their body for anatomical study. Only $47 \%$ of the participants were willing to campaign for body donation while $53 \%$ of the participants were not willing to campaign. $56 \%$ of the participants agreed that it is noble to donate one's body after death for anatomical study/research. Only $23 \%$ of the participants agreed to consider body donation while $77 \%$ of the participants said they would not (figure 4).

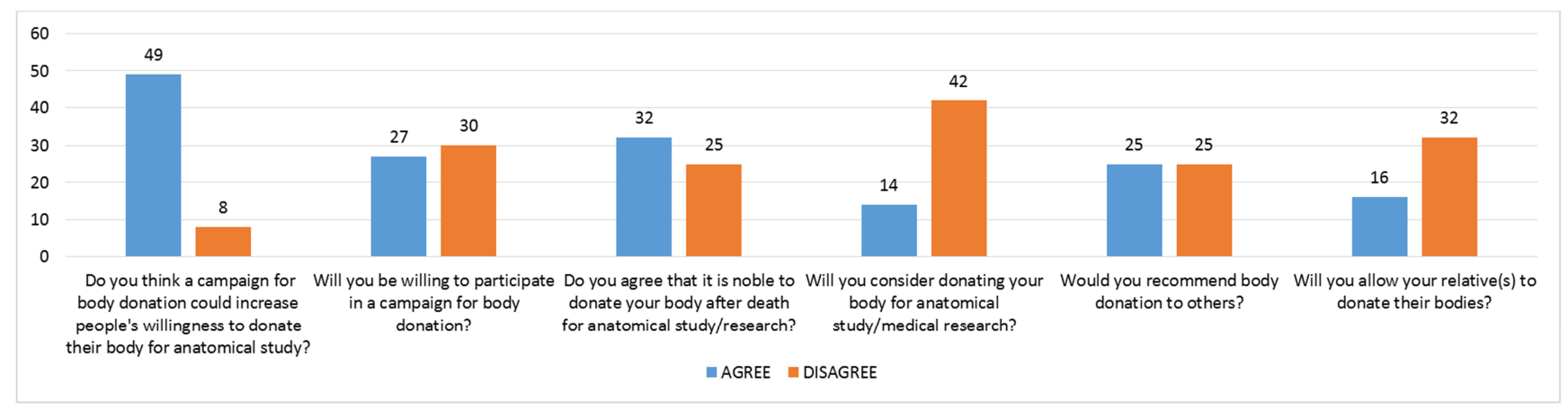

Figure 4. Evaluation of participants' willingness to free will body donation for anatomical study. 


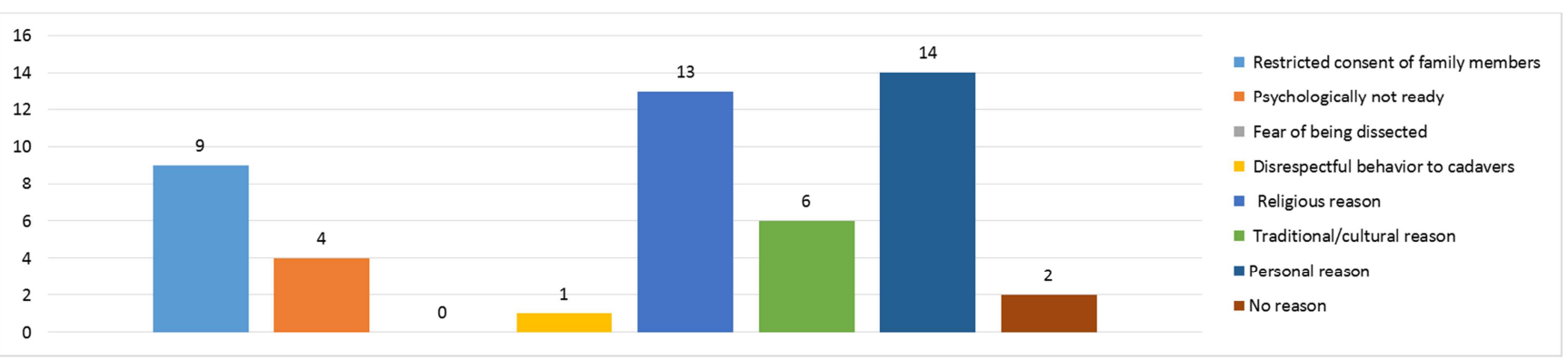

Figure 5. Showing reasons for participants' unwillingness towards body donation.

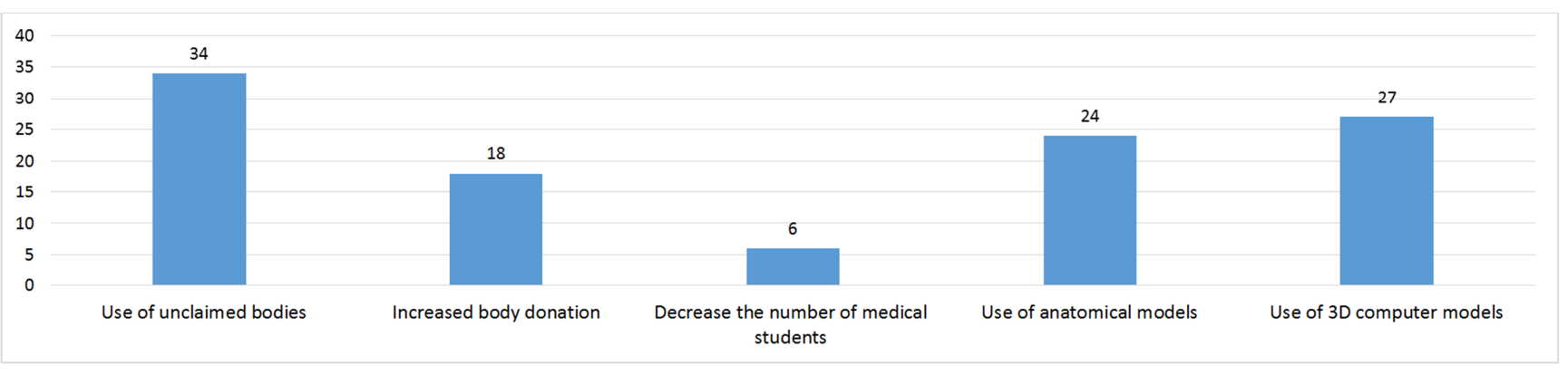

Figure 6. Showing participants suggestions on how to solve cadaver insufficiency in Nigeria.

\section{Discussion}

The use of Cadaveric dissections has over the years been a fundamental tool for the study of anatomy and has proven to be the most reliable tools in the study of anatomy [4]. and dissection has been considered as an essential requirement in learning gross anatomy particularly the three-dimensional aspect of human anatomy and has remained a major requirement for medical and biomedical studies $[1,3,17$, 21]. However, due to the increase in medical schools in Nigeria, it has been quite difficult to balance the cadaver to student ratio, as the major source of supply for cadavers is from condemned criminals and unclaimed bodies which are no longer able to satisfy the ever growing need of cadavers for pedagogic purposes in medical institutions in Nigeria, hence the purpose of this study which is tailored towards evaluating how Nigerians view body donation as a valid alternative to the use of unclaimed bodies for anatomical teaching. This Lack of knowledge of body donation is a barrier to becoming active donors.

The results in this research showed that with the participants' level of knowledge, most (85.96\%) affirmed that a campaign on body donation would increase people's willingness to donate their bodies for anatomical study. Some (47.4\%) were willing to participate in the campaign and they thought it as noble to donate one's body for anatomical study (56.1\%).

Surprisingly, only a few of the participants $(25 \%)$ were willing to donate their bodies for anatomical study. Their unwillingness was mainly due to religious reasons (26.5\%), culture and tradition and also restrictions from family members. Some even had reasons they would not disclose (28.5\%). They could recommend body donation to others but will not recommend it to their relatives.
Possible reasons for participants' unwillingness to body donation were; restricted consent from family members, psychologically not ready, the fear of being dissected, disrespectful behavior towards cadavers, religious, traditional and cultural reasons.

This research shows that the participants accept body donation as a way of solving scarcity of cadaver supply. However, they would not donate their bodies for anatomical study and would not recommend it to their relatives but would recommend it to others.

\section{Conclusion}

The supply of cadaveric specimen for anatomical education is gradually becoming a thing of concern hence the need for informative and practical methods that will aid in combating this shortage. Body donation campaigns leading to body donation programs as shown in this study is now a necessity in Nigeria. The results in this study suggest that the idea of body donation is well known in Nigeria especially among anatomists but the major challenge as shown is the willingness to campaign, donate and recommend body donation to friends and family. Religious beliefs and many other undisclosed reasons might be the cause of this major challenge. Body donation and bequest programs will serve as a major tool in the attempt to curb the shortage in supply of cadaveric specimen both now and the future.

\section{References}

[1] Akinola OB. Formal body bequest program in Nigerian medical schools: When do we start?. Anatomical sciences education. $2011 \mathrm{Jul} 1 ; 4$ (4): 239-42. 
[2] Azis, M. A., J. C. Mckenzie, J. S. Wilson, R. J. Cowie, S. A. Ayeni and B. K. Dunn, 2002. The human cadaver in the age of biomedical informatics. Anat. Rec., 269 (1): 20-32.

[3] Bolt, S., Venbrux, E., Eisinga, R., Kuks, Veening, J. B. M., Gerrits, P. O. (2010). Motivation for body donation to science: more than altruistic act. Annals of anatomy. 192 (2), 70-74.

[4] Delmas, V. (2001). Donation of bodies to science. Bull academy: Natl med. 185: 849-856. education: knowledge is the ultimate gift of body donors. Singap. Med. J., 48 (3): 186190.

[5] Ellis, H., 2001. Teaching in the dissecting room. Clin. Anat., 14: $149-151$.

[6] Gangata H, Ntaba P, Akol P, Louw G. 2010. The reliance on unclaimed cadavers for anatomical teaching by medical schools in Africa. Anat Sci Educ 3: 172-183.

[7] Jones, D. G., 1997. Reassessing the importance of dissection: A critique and elaboration. Clin. A nat., 10: 123-127.

[8] Lazarus. L. Sookrajh, R. \& Satyapal, K. S. (2014). A historical perspective of anatomical pedagogy at university of KwaZuluNatal: a pilot study. South Africa.

[9] Mandel, S., Gupta, A. \& Chatter Jee, M. (2015). Bodies do matter: rethinking anatomical pedagogy, from the scalpel to the mouse: a review in international journal of anatomy and research. Vol3 (4). 1559-67. ISSN2321-4287. DOI: http/dx.dox.org, 10.16965/ijar.

[10] Mwachaka, M. P. \& Saidi. H. (2016). Repeated exposure to dissection does not affect student's attitude towards human body for Anatomy teaching.

[11] Odebode N. 2008. Lagos coroner's law: 12 pathologists contend with 40 corpses daily. 27 August 2008. The Punch Newspaper, Ikeja, Lagos, Nigeria. URL: http://www.punchng.com/Articl.aspx?theartic=Art200808271 443928 [accessed 22 july 2019].
[12] Older, J., 2004. Anatomy: A must for teaching the next generation. Surg. J. R. Coll. Surg. Edinb. Irel., pp: 79-90.

[13] Osuagwu, F. C, Imosemi, I. O \& Oladejo, O. W. (2004) Sources of cadaver use for dissection at the Ibadan medical school, Nigeria- analysis of a 3-year data. African journal of biomedical research. Vol 7; 93-95. ISSN 1119-5096. Ibadan biomedical communication group.

[14] Izunya AM, Oaikhena GA, Nwaopara AO. 2010. Attitudes to cadaver dissection in a Nigerian medical school. Asian J Med Sci 2: 89-94.

[15] Rajkumari, A. B. and Y. I. Singh, (2007). Body donation and its relevance in anatomy learning - A review. J. Anat. Soc. India, 56 (1): 1-6.

[16] Riederer B. M. (2016), Body donations today and tomorrow: What is the best practice and why? Clin. Anat., 29: 11-18. Doi: $10.1002 /$ ca. 22641

[17] Rizzolo. L. J. (2002). Human dissection: an approach to interweaving the traditional and humanistic goals of medical education; anatomy record 269: 242-248.

[18] Roach, M. (2004). stiff: the curious lives of human cadavers.

[19] Rokade SA, Bahetee BH (2013). Body donation, A review. Medical journal of western India. 41 (1): 36-40

[20] Savulescu, J. (2003). Death, us and other bodies: personal reflections. Journal of medical ethics, 29 (3), 127-130.

[21] Sehirli US, Saka E, Sarikaya O. 2004. Attitudes of Turkish anatomists toward cadaver donation. Clin Anat 17: 677-681.

[22] Slotruck, H. B. \& Hilton, S. R. (2006). Proto- professionalism and the dissecting laboratory, clinical anatomy; 19: 429-436. 